CARNETS OE Carnets de géographes

GÉOGRAPHES.

Géographies critiques

\title{
La voix des chercheur(-e)s et la parole du migrant
}

Ce que les coulisses du terrain maltais nous enseignent

Nathalie Bernardie-Tahir et Camille Schmoll

\section{(2) OpenEdition}

Journals

Édition électronique

URL : http://journals.openedition.org/cdg/1000

DOI : $10.4000 /$ cdg. 1000

ISSN : 2107-7266

Éditeur

UMR 245 - CESSMA

Référence électronique

Nathalie Bernardie-Tahir et Camille Schmoll, « La voix des chercheur(-e)s et la parole du migrant »,

Carnets de géographes [En ligne], 4 | 2012, mis en ligne le 01 septembre 2012, consulté le 21 avril 2019.

URL : http://journals.openedition.org/cdg/1000; DOI : 10.4000/cdg.1000

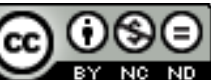

La revue Carnets de géographes est mise à disposition selon les termes de la Licence Creative Commons Attribution - Pas d'Utilisation Commerciale - Pas de Modification 4.0 International. 


\title{
LA VOIX DES CHERCHEUR(-E)S ET LA PAROLE DU MIGRANT
}

Ce que les coulisses du terrain maltais nous enseignent

NATHALIE BERNARDIE-TAHIR

Université de Limoges

UMR Géolab

$\underline{\text { nathalie.bernardie-tahir@unilim.fr }}$

\author{
CAMILLE SCHMOLL \\ Université Paris-Diderot \\ UMR Géographie-cités / CEDREF \\ camilleschmoll@yahoo.fr
}

\section{Chercheur(-e)s critiques : un oxymore ?}

Objectivité et engagement font partie de ces couples d'oxymores qui ont longtemps déterminé les structures fondamentales de la recherche. En sciences sociales tout particulièrement, un bon chercheur devait avant tout n'être "d'aucun temps ni d'aucun pays " pour paraphraser la formule de Fénelon, la neutralité étant considérée comme garante de la qualité scientifique. En creux, l'engagement, expression d'une subjectivité empreinte de valeurs (et non de faits) signait l'échec de la fabrique scientifique. C'est du moins un postulat qui a durablement imprégné les démarches positivistes, néo-positivistes et modernes des $X X^{e}$ et $X X^{e}$ siècles, même si dans le détail, Isabelle Lefort, en évoquant notamment la figure de l'anarchiste et non moins géographe Elisée Reclus, rappelle qu'« expulsées par la grande porte du dessein disciplinaire, les valeurs morales ou esthétiques sont toujours rentrées par les fenêtres et autres interstices, sous la forme de vents coulis ou d'ombre portée " (Lefort, 2010, p.13). L'introduction de la valeur, de l'éthique et de la subjectivité dans la démarche scientifique s'est opérée au cours des dernières décennies dans la recherche anglosaxonne, au fondement des études postmodernes, postcoloniales et subalternes qui s'épanouirent dans la littérature britannique et américaine à partir des années 1970. 
Les interdits jusque-là imposés par la doxa de l'objectivité rationnelle furent évacués, remplacés par la nécessité d'une démarche réflexive, posant clairement la question du positionnement et de la positionnalité du chercheur, assumant pleinement sa nonneutralité et ses choix, qu'ils soient relatifs au sujet retenu, aux groupes étudiés, aux lieux de l'enquête, à la finalité et aux modalités de restitution de la recherche (Milhaud, 2006). Désormais écrits à la première personne du singulier, ces travaux émanant pour l'essentiel d'anthropologues, d'ethnologues ou de sociologues traduisent la posture de chercheurs libérés de l'académisme de l'objectivation scientifique.

En géographie toutefois, et surtout dans la géographie française, les approches développant une réflexivité autour de la construction de l'objet et du positionnement du chercheur furent globalement plus tardives et plus longues à s'imposer, même si elles se multiplient depuis ces dernières années sous la plume de jeunes géographes plaçant le questionnement éthique au cœur de leurs travaux (Brachet, Cruse, Morelle, Milhaud, Ripoll, Zeneidi, etc.). II est en effet encore de bon ton et très courant d'objectiver la démarche de recherche, à la différence de ce qui est pratiqué dans d'autres sciences sociales, si bien que les coulisses, les émotions (Widdowfield, 2000), les bricolages et autres petits arrangements qui forment le quotidien des démarches qualitatives sont souvent effacés voire dissimulés au moment de la restitution de la recherche. Or les géographes, et plus particulièrement ceux qui travaillent sur des thèmes impliquant directement des relations de pouvoir et de domination, ne peuvent ni faire l'économie d'une posture critique, tant il est vrai que le choix même de travailler sur telle ou telle question dénote a priori un engagement et une conception particulière de la recherche, ni échapper à une réflexion sur les "coulisses de la recherche " qui revêt nous semble-t-il deux enjeux majeurs.

Le premier défi à relever consiste à opérer un savant " dosage » entre engagement et distanciation, une véritable gageure tant la marge est étroite entre le risque d'une "surinterprétation dénonciatrice" (Milhaud, 2006) que peuvent faire courir une empathie et un engagement trop marqués à l'égard des groupes sociaux étudiés, et celui d'une interprétation faussée et caricaturale qu'une posture distanciée ou " extérieure » peut produire. C'est pourquoi il nous paraît si important de lever le voile sur "cette petite cuisine interne", sur les ajustements et rééquilibrages constants auxquels le chercheur doit procéder sur le terrain pour éviter ces deux écueils, et qui sont d'autant plus complexes à décrire et à saisir qu'ils varient selon les objets ou les moments de la recherche, mais aussi selon la personnalité même des chercheurs.

Le deuxième enjeu est directement lié au premier et concerne le rapport objet/sujet. Car c'est bien sur le terrain que la question foucaldienne de l'objectivation et de la subjectivation se pose avec le plus d'acuité : "l'objet de recherche » pensé au départ de manière théorique correspond dans la réalité à des hommes et des femmes qui agissent, décident, parlent, imaginent, construisent, projettent, choisissent, bref, à des 
sujets, souvent relégués d'ailleurs au rang d'objet par le discours normatif dominant. Le terrain est donc le lieu où ces deux conceptions s'affrontent et où le chercheur étudie un objet tout en créant dans le même temps des relations personnelles plus ou moins fortes avec des individus-sujets. Dans ce contexte, il est donc particulièrement nécessaire de connaître et de faire connaître ces oscillations qui, dans les coulisses du terrain, bousculent en permanence la place relative du chercheur et des populations enquêtées en faisant bouger les lignes de l'autorité narrative.

Aussi, dans ce texte, nous souhaitons raconter comment nous nous sommes emparées de la problématique des migrations dites "irrégulières » à Malte, en mettant l'accent sur le caractère chaotique et accidenté de nos trajectoires de recherche et de notre expérience de terrain. Dans les périphéries sud de l'Europe, les îles forment depuis longtemps des lieux emblématiques de la dynamique migratoire européenne. Ces lieux que I'on quittait autrefois pour survivre sont aujourd'hui devenus des territoires d'accueil, à la confluence des flux de migrations régulières et irrégulières. Ces dernières, extraordinairement médiatisées et présentées le plus souvent de manière fort caricaturale, interpellent aujourd'hui les instances politiques comme l'opinion publique européennes, alors même que leurs contours restent sujets à caution.

A Malte, c'est au début de la décennie 2000 que l'on commença à enregistrer ce type de flux migratoires en provenance d'Afrique du Nord, d'Europe centrale et orientale, d'Asie et d'Afrique sub-saharienne. Une partie de cette immigration s'effectue par voie maritime, depuis l'Afrique du Nord: localement qualifiée de "clandestine " ${ }^{1}$, (klandestina en maltais), elle a débuté en 2002 avec l'arrivée de 1686 migrants. Depuis lors, on a pu comptabiliser 12500 arrivées sur les côtes maltaises. Si cette migration est numériquement minoritaire, elle représente pour Malte comme pour l'Europe un véritable enjeu social et politique. Malte, petit Etat à la densité exceptionnelle, est devenue en effet l'un des bastions de ce qu'il est coutume d'appeler dans le langage courant, la "forteresse Europe », tout à la fois île barrière et lieu de matérialisation du rapport d'altérité de l'Europe avec le reste du monde.

C'est sur ces migrants "indésirables" (Agier, 2008) qui font l'objet de toutes les stigmatisations et de tous les efforts de l'Union Européenne dans son entreprise de renforcement des frontières, que nos enquêtes se sont focalisées. Nos questionnements, multiscalaires, portaient tout autant sur la place de l'île dans les

\footnotetext{
${ }^{1}$ Rien n'est plus confus que la typologie des migrants qui est établie aujourd'hui. Les oppositions entre migrations régulières et irrégulières ou "non documentées " (Collyer, 2006, 2010) ne sont pas toujours très pertinentes tant la porosité peut être forte entre les deux. Un migrant peut entrer régulièrement sur un territoire, mais devenir irrégulier s'il reste au-delà de l'expiration de son permis de séjour. Il s'agit d'ailleurs de la principale forme d'immigration irrégulière recensée en Europe, bien que la moins visible. A l'inverse, un migrant arrivé sans titre de séjour peut rapidement se trouver en situation régulière s'il demande l'asile ou s'il obtient un statut de protection. A Malte où les instances politiques reconnaissent et appliquent la convention de Genève, près de la moitié des migrants surnommés " klandestini " sont des demandeurs d'asile ou bénéficient d'un statut de protection (réfugié, protection subsidiaire, protection humanitaire, etc.) et sont donc « documentés », en situation régulière.
} 
trajectoires migratoires et sur son rôle dans la construction d'une image de l'Europe ${ }^{2}$, que sur les modalités politiques de management et de gestion des flux à toutes les échelles, sur les formes d'installation des migrants dans l'île ainsi que sur la transformation des rapports et identités de genre en migration. C'est au cours de notre travail de terrain ${ }^{3}$ que la question de notre positionnement et de notre rapport aux migrants s'est posée, voire s'est imposée à nous.

Face à une situation d'urgence que nous n'imaginions pas, le souci éthique s'est immédiatement heurté aux considérations strictement scientifiques et la problématique théorique de l'engagement et de la neutralité dans la recherche a soudain pris une tournure très concrète: comment dénoncer - car dénoncer apparaissait comme une évidence - sans tomber dans un militantisme trop éloigné des contingences scientifiques ? Comment analyser finement et sereinement une situation marquée par une si forte tension entre tous les acteurs de la scène migratoire, migrants, chercheurs, managers de centre d'hébergement ou de rétention, fonctionnaires gouvernementaux, responsables et employés d'ONG, médiateurs culturels, etc. ?

Durant chacune de nos missions nous nous sommes efforcées de ne pas mélanger les registres, mais le plus souvent vainement comme nous allons le montrer. Parallèlement, la volonté de réagir, d'intervenir, de "faire quelque chose » pour améliorer le sort des migrants nous a renvoyées à un autre écueil : celui d'enfermer ces individus dans une posture de victimes, d'individus dé-responsabilisés qui ne feraient que subir les contraintes d'une situation qu'ils n'ont pas choisie, et donc de les reléguer au rang d'objets. Or, dans la lignée de l'ethnométhodologie ${ }^{4}$ et des subaltern studies, nous souhaitions précisément leur donner la parole, montrer leur capacité de résistance et d'autonomisation en mettant en évidence les multiples formes de tactiques et de stratégies, individuelles et collectives, mises en place pour améliorer leur situation au quotidien et, plus largement, en leur restituant la possibilité d'être narrateurs de leur propre histoire. Comment donc parvenir à défendre des personnes vulnérabilisées et exposées à de nombreuses formes d'injustice sans tomber dans le piège de la victimisation, si fréquente dans les études sur les réfugiés et les

\footnotetext{
${ }^{2}$ Dans le cadre du Programme Eurobroadmap qui a bénéficié d'un soutien financier du septième programme-cadre de la Communauté européenne (PC/2007-2013) en vertu de la convention de subvention no 225260 . Voir notamment Anna Spiteri, Camille Schmoll, et Maurice Saïd, 2011, "Somali women in Malta», Final report of WP3 Visions of Migrants, working paper disponible sur www.eurobroadmap.eu.

${ }^{3}$ Nous avons effectué plusieurs séjours de recherche sur l'île de Malte, mêlant observations, entretiens auprès des migrants et rencontres avec des acteurs-clef de la scène migratoire maltaise (travailleurs sociaux, responsables d'ONG, membres du gouvernement, gérants de centres). Nous avons effectué un séjour de recherche commun en mai 2011, à Hal Far et Balzan. Auparavant, Nathalie Bernardie-Tahir avait réalisé deux missions, l'une en février 2010, avec Anne Blanchier, l'autre en avril 2010, en compagnie de la photographe Elizabeth Cosimi, dans le cadre du programme Miriade (cf. http://www.flsh.unilim.fr/miriade). Ses observations se sont concentrées sur les centres de Hal Far et de Marsa. Les deux premiers séjours de recherche de Camille Schmoll ont eu lieu en mai 2010, en compagnie d'Anna Spiteri, et en octobre 2010, dans les centres d'hébergement de Hal Far et de Balzan.

${ }^{4}$ Pour une application de l'ethnométhodologie en géographie, voir Staszak, 1997
} 
demandeurs d'asile? Finalement, comment le chercheur peut-il concilier son engagement, dans un souci éthique et citoyen, et l'effacement relatif de son autorité narrative, sans en rabattre sur ses exigences scientifiques?

$C^{\prime}$ est ce mouvement de balancier constant entre voies de traverse militantes et travail académique, entre pas de côté, mouvements de décentrement et de recentrage, jeux de distance et d'engagement, processus d'objectivation et de subjectivation que nous souhaitons ici raconter, sans en tirer de conclusions à portée générale, mais en montrant simplement comment nous avons géré ces oscillations, qui ont à la fois généré des remises en cause profondes et constitué des temps forts de notre recherche sur le terrain. Nous restituerons notre cheminement par un parcours en deux temps, celui de l'engagement et de la dénonciation, d'une part, et celui de la mise en lumière des subjectivités et résistances migrantes, d'autre part. Une telle distinction est nécessaire pour la clarté de l'exposé, même si elle ne rend pas suffisamment compte des complémentarités entre ces deux phases et du "brouillage " des voix qui s'opère lors du processus de recherche, comme nous le soulignerons en conclusion.

\section{1. « Faire quelque chose " ou travailler en terrain d'urgence ...}

Commençons par préciser ici que cette recherche sur les migrations dites "irrégulières " à Malte constituait pour nous une nouveauté, ayant respectivement travaillé jusqu'alors sur les migrations économiques en Italie et sur les questions de développement dans divers territoires insulaires. Ainsi, lorsque nous arrivâmes à Malte en 2010, après avoir élaboré au préalable un protocole de recherche et pris divers contacts locaux avec des personnes-ressources, ni l'une ni l'autre n'étions réellement "préparées " à faire face à la situation d'urgence dans laquelle les migrants étaient plongés, ni à entendre cette longue liste d'histoires personnelles toutes plus dramatiques les unes que les autres. En un mot : ce fut un choc, comme l'illustrent ces morceaux choisis de récits :

" Je suis de Mogadiscio où je vivais avec mes parents, mes trois frères et ma sœur. Mon père tenait un atelier de réparation mécanique. Un jour mon frère a reçu une balle alors qu'il marchait dans la rue et s'est retrouvé gravement handicapé. Mes parents m'ont alors incité à partir. Je ne voulais pas, je voulais rester avec eux et avec ma femme, mais ma mère m'a dit que je ne pourrais pas les aider en restant. Alors je suis parti, c'était le 2 avril 2008. J'ai traversé la Somalie, l'Ethiopie, le Soudan, le désert jusqu'en Libye (soit un périple de plus de $6000 \mathrm{~km}$ ). Puis j'ai tenté de traverser la Méditerranée une première fois pour gagner l'Italie. Nous sommes tombés en panne de fuel, nous avons alors dérivé pendant plusieurs jours, la moitié des hommes du bateau sont morts de soif et nous avons dû les jeter par dessus bord. Finalement, les courants nous ont rabattus vers les côtes tunisiennes où les survivants ont été 
secourus. Je suis resté entre la vie et la mort pendant trois semaines à l'hôpital. Quelque temps plus tard, je suis reparti en Libye et j'ai retenté ma chance en mer. Notre bateau a été repéré par des garde-côtes maltais et nous avons été placés en centre de détention. J'y suis resté emprisonné un an, puis on m'a emmené ici, au hangar ». Souleiman, Somalien, 24 ans, Hal Far Hangar. 2010

" Je suis partie d'Erythrée avec mon mari. Nous avons traversé le désert jusqu'en Libye où nous sommes restés quelques mois afin de trouver l'argent pour traverser. J'étais enceinte de 5 mois lorsque nous avons trouvé un bateau, mais nous n'avons pas pu embarquer sur le même. Le voyage a duré 5 jours (contre 2 prévus normalement), c'était très dur et les gens avaient très peur. Finalement les autorités maltaises nous ont interceptés et j'ai été détenue dans le centre fermé de Ta'Kandja. Le bateau de mon mari n'est jamais arrivé. Je suis ici toute seule, et je vais bientôt accoucher ». Froweni, Erythréenne, 19 ans, Hal Far centre pour famille. 2010

"Nous avons quitté la Libye dans l'urgence (en avril 2011), car des centaines d'Africains sont morts, tués par les rebelles qui nous prenaient pour des mercenaires à la botte de Kadhafi. On est monté dans un bateau avec mon mari et mes deux jumeaux de 1 an et 2 mois. Nous avons dérivé pendant 10 jours, un hélicoptère est passé et ne s'est pas arrêté, un bateau de l'OTAN nous a vus mais n'a pas donné l'alerte. J'ai donné du dentifrice à mes bébés pour qu'ils aient quelque chose à manger. Nous sommes ici dans ce hangar depuis 1 mois ». Djuma, Erythréenne, Hal Far. 2011.

Les histoires que les migrants racontent ici sont terribles. Tous ont surmonté le déracinement du départ, l'angoisse de l'inconnu, survécu au périlleux passage en Méditerranée, mais aussi à la faim, à la soif et à l'épuisement lors de la longue traversée du désert soudano-libyen, aux tabassages, rackets et viols perpétrés par les militaires ou policiers patrouillant dans le désert ou à Tripoli.

A Malte, les conditions de vie des migrants, dans ou hors des centres, ne sont en outre guère plus acceptables ${ }^{5}$. Dès notre arrivée au centre de Marsa, des migrants ont tenu à nous conduire dans une chambre où venait de se suicider l'un des leurs, à bout de nerf et d'espoir, ne supportant plus ses conditions de vie et les brimades quotidiennes. Dans le hangar d'Hal Far, la situation déjà intolérable au début de l'année 2010 s'est dégradée au fil des mois au point de devenir parfaitement insupportable un an plus tard. Dans cet imposant bâtiment blanc couvert de tôles, près de 200 lits superposés se répartissaient sur la grande dalle carrée de béton abîmé. En cette fin d'hiver 2010, quatre cents hommes s'entassaient dans ce lieu sombre et sale en lieu et place des anciens avions de la British Royal Air Force. A l'extérieur, tout était aussi indécent qu'à I'intérieur. Le dispositif sanitaire consistait en une trentaine de cabines individuelles en plastique grises et bleues servant de douche ou de WC pour les 600 personnes du

\footnotetext{
${ }^{5}$ Il existe à Malte deux types de centres : les centres fermés, dans lesquels les migrants peuvent être détenus à leur arrivée sur l'île pour une durée maximale de 18 mois, et les centres ouverts, qui hébergent les migrants à leur sortie de détention dans des conditions semi-carcérales.
} 
centre. Disposée le long d'un des murs du bâtiment en chantier, une rangée d'éviers crasseux abritait le " coin cuisine ", nom très abusif donné à un petit espace d'environ $15 \mathrm{~m}^{2}$, percé d'ouvertures sans portes ni fenêtres où les migrants pouvaient faire cuire leurs repas sur une dizaine de feux de cuisson installés sur un support en béton d'une saleté repoussante. Ici et là, les violentes averses des semaines précédentes avaient laissé d'immenses flaques se transformant en cloaques où surnageaient boîtes de conserve, paquets de cigarettes et emballages en plastique.

Pourtant, la réalité du hangar d'Hal Far, déjà très choquante au printemps 2010, dépassa l'entendement un an plus tard, en pleine "crise libyenne ». Fuyant les persécutions en Libye, des centaines de migrants dont des familles entières avec enfants étaient arrivées à Malte, rapidement transférées dans le hangar dans lequel 30 tentes de la Croix Rouge avaient été disposées, remplaçant le dispositif de l'année précédente. Les conditions sanitaires étaient si épouvantables que les enfants, dont la plupart étaient en bas âge, tombaient malades les uns après les autres, certains ayant même cessé de s'alimenter et de boire. En cause les émanations d'hydrocarbure remontant du sol, la multiplication de germes résistants qui se traduisaient par de fortes fièvres, vomissements et diarrhées à répétition. Désemparés et craignant pour la vie de leurs enfants, les parents nous avaient livré leurs angoisses, souhaitant même pour certains retourner en détention pour bénéficier de meilleures conditions.

" J'ai fui la Libye avec mon mari et mes deux jumeaux. Ils ont un an et deux mois. La traversée a été horrible, nous avons dérivé pendant 10 jours avant d'être secourus. Je donnais du dentifrice à mes enfants pour qu'ils mangent quelque chose. Nous sommes arrivés épuisés. Et où est-ce qu'on nous met? Dans ce hangar sale et dangereux ». Djuma, Erythréenne.

"J'habitais à Zaouia (Libye) depuis quatre ans, avec ma femme et mon fils Miki qui a un an et demi. J'étais professeur d'anglais là-bas. Les rebelles (en Libye) ont tué des centaines d'Africains, dont beaucoup d'amis à moi. J'ai fermé la porte de ma maison et suis parti en bateau avec ma femme et mon fils, avec 260 personnes à bord. On a eu de la chance, le voyage n'a duré que 34h, on est arrivé à Malte le 29 mars (2011), puis dans ce hangar le 19 avril. Mon fils est tombé malade peu après, il a de la fièvre en permanence et mange peu. Les surfaces sont huileuses, les évacuations d'eau sont défectueuses, des rats courent partout. Tout cela est toxique. Les bébés marchent à quatre pates sur le sol, ils se touchent la bouche, les yeux, ils ont des infections, ils sont malades. Mon fils a une conjonctivite, il tousse, il vomit, il a la diarrhée. Il est sous antibiotique, mais il faiblit, il perd du poids. Tous les enfants sont dans le même état. Il faut les emmener tout le temps à l'hôpital. Nous avons vu un médecin italien pleurer en les voyant. Elle répétait : Que puis-je faire, que puis-je faire? ». Dawit, 35 ans, Ethiopien. 
Carnets de géographes, $\mathrm{n}$ ㅇ 4, septembre 2012

Rubrique Carnets de terrain

Figure 1 : Le Hangar (Hal Far) : mars 2010
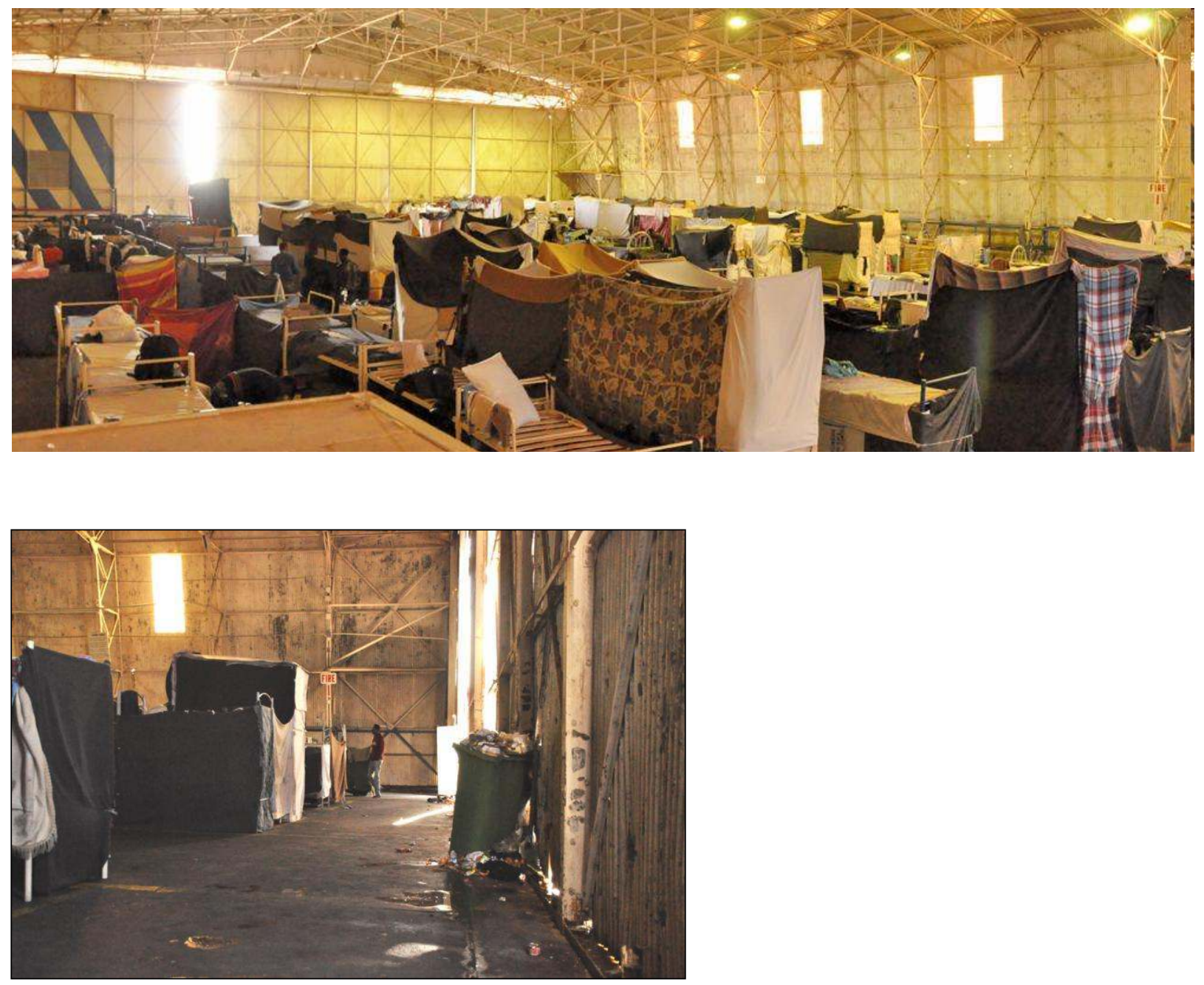

Figure 2 : Le Hangar (Hal Far) : mai 2011

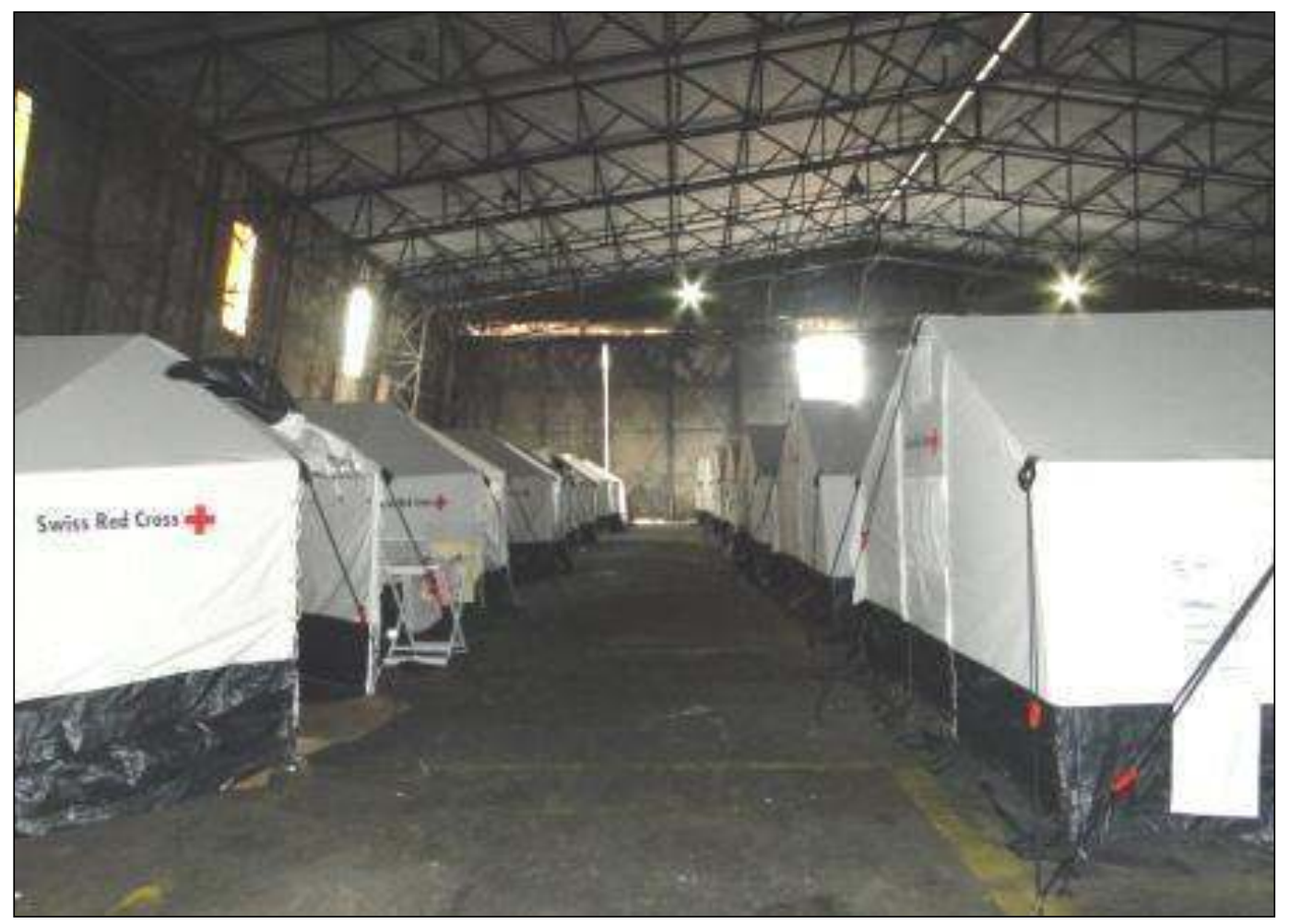


Parties pour mener des entretiens dans le cadre d'un protocole de recherche rigoureux, nous nous sommes trouvées immédiatement plongées dans un tourbillon émotionnel, où la compassion et le sentiment d'injustice le disputaient à la colère et à la tristesse. Car c'est une chose de savoir que la situation des migrants à Malte est difficile, c'en est une autre de voir les lieux et les hommes, de sentir le renfermé dans la promiscuité des chambres, d'être incommodé par la puanteur des poubelles qui côtoient les lits dans le hangar. C'en est une autre aussi de regarder des visages dévastés par l'angoisse, d'entendre la parole de ces rescapés et, pire, leurs silences sur l'indicible, de percevoir leur stress dans une voix trop forte ou dans des gestes compulsifs, de deviner leur désespoir contenu et leur détermination vaciller, d'entendre un rire d'enfant résonner dans cet univers quasi carcéral. Le " terrain » se vide peu à peu de sa dimension théorique et prend chair, s'incarne dans la proximité corporelle des migrants et des chercheur-es. L'information passe moins par l'esprit et la parole que par le corps et les sens en alerte qui captent et produisent un autre langage, dominé par l'émotionnel et le subjectif. Cette relation physique, écrit Marie Morelle, place les chercheur-es "en situation d'imprévisibilité radicale " (Morelle, 2009, p. 159), et explique la réaction immédiate qui est celle de s'impliquer, de dénoncer, de "faire quelque chose", par altruisme bien sûr, mais aussi comme catharsis permettant d'évacuer un "trop-plein émotionnel » que la seule prise de notes dans un carnet ne suffit plus à purger. Mais que faire, comment ?

Nous voici donc renvoyées au dilemme originel: observer et analyser tout en conservant cette neutralité axiologique chère à Max Weber, au risque de passer pour des voyeurs, voire d'être accusés de non-assistance à personne en danger, ou dénoncer et s'engager, au risque de perdre toute forme de distanciation critique. Mais au fond est-ce bien un dilemme ? Faut-il continuer à penser recherche objective et approche militante comme deux inconciliables ? L'engagement n'est-il pas soluble dans la démarche scientifique ? Les migrants du centre de Marsa ont réactivé cette problématique quand, après plusieurs heures d'entretiens, ils nous ont pris à partie en nous accusant de voler leurs histoires, de nous en servir à des fins personnelles alors que rien ne change pour eux qui restent là, dans ce centre.

A l'urgence de la situation, notre réflexe a donc été de répondre par l'urgence de la dénonciation en proposant deux articles, non pas dans des revues scientifiques mais dans la presse écrite, caressant l'espoir de faire bouger les lignes en alertant l'opinion publique. Ces publications dans le Monde Diplomatique ${ }^{6}$ et dans Mediapart ${ }^{7}$ nous permettaient ainsi non seulement "d'agir » en révélant l'inhumanité des conditions de vie des migrants retenus à Malte tout en apaisant notre révolte intérieure, mais aussi

\footnotetext{
6 "Malte l'île-prison », Nathalie Bernardie-Tahir et Anne Blanchier, Les blogs du diplo, Le Monde Diplomatique, 08/11/2010 - http://blog.mondediplo.net/2010-11-08-Malte-l-ile-prison

${ }^{7}$ "Réfugiés de Libye : les oubliés de Malte », Carine Fouteau, Mediapart, 08/06/2011.

http://www.mediapart.fr/journal/international/070611/refugies-de-libye-les-oublies-de-malte
} 
de porter la critique et la dénonciation dans un espace dédié : celui du journalisme. Si elles furent pour nous la solution du moment, ces initiatives spontanées nous attirèrent parallèlement des problèmes. N'ayant que très modérément apprécié le contenu de l'article intitulé Malte, l'île-prison, le responsable des centres ouverts de Malte décida de nous refuser l'autorisation d'entrer dans les centres, un véritable obstacle pour notre travail de terrain. La confrontation entre travail de recherche et engagement est ainsi complexe mais souvent incontournable, se traduisant par des formes de conciliation possibles mais aussi par des interférences imprévisibles et problématiques ${ }^{8}$.

Ceci dit, si la dénonciation peut conduire comme ici à fermer certaines portes ${ }^{9}$, elle met également à jour des mécanismes subtils de régulation de la part des autorités sur l'accès à l'information ${ }^{10}$. Ainsi, nous apprenions à nos dépens qu'il y a pour le gouvernement des lignes rouges à ne pas franchir si l'on souhaite accéder aux lieux d'hébergement des migrants (voir aussi Zeneidi, 2010). Les contours de ces lignes sont flous et évoluent: l'ouverture et la fermeture des centres d'accueil dépend des opportunités politiques du moment et est le fruit d'équilibrages entre volonté d'attirer l'attention de l'opinion européenne ou, au contraire, de cacher la brutalité de l'accueil réservé aux migrants, choix de mise en visibilité de lieux et de situations " modèles " " et dissimulation des situations les plus critiques. Tout cela enrobé d'une rhétorique de l'urgence et de la lutte face à la menace d'invasion, qui tend à priver de toute légitimité la possibilité d'expression d'une voix discordante sur la situation migratoire de l'île.

\section{2. ... Mais éviter la victimisation}

La dénonciation apparaît comme une étape forte et importante du processus de recherche. Elle est certainement valorisante (et parfois apaisante) pour le chercheur, elle est parfois porteuse de résultats concrets, elle permet également de faire apparaître certains mécanismes de domination au cœur des dynamiques migratoires ainsi que les ressorts subtils du contrôle de l'information. Pour autant elle ne suffit pas. La dénonciation, parce qu'elle s'attache à mettre en lumière des situations d'injustice

\footnotetext{
${ }^{8}$ Notre démarche s'apparente à ce que les éthnométhodologues nomment le breaching (de Luze, 1997): émettre des provocations, introduire une brèche dans la situation observée pour mettre à jour les normes sociales et, en l'occurrence, des mécanismes ordinaires de contrôle et de domination.

${ }^{9}$ Ceci explique pourquoi le second article dans Mediapart a été écrit par une journaliste, Carine Fouteau, à qui nous avions relaté dans le détail la situation dans le hangar en mai 2011. Ayant prévu d'organiser un colloque international sur la question des migrations irrégulières à Malte en décembre 2011 (http://www.flsh.unilim.fr/miriade), nous avons préféré rester dans l'anonymat de manière à ne pas compromettre la tenue de cette manifestation scientifique. C'est pourquoi nos noms n'apparaissent pas dans l'article sur "les oubliés de Malte ".

${ }^{10}$ La pratique est courante : à Malte une autre chercheuse en sciences sociales s'est vue refuser l'accès à tous les centres d'hébergements pour migrants pour avoir posté un billet sur un blog.
} 
et d'urgence, s'embarrasse difficilement de la diversité des situations qui composent les mondes migratoires. Parce qu'elle s'attache à décrire la vulnérabilité des individus, elle porte en elle le risque de réduire l'autre en victime, en "sans voix". Or les migrants, tout opprimés qu'ils peuvent être, sont porteurs de voix multiples, mais aussi de multiples actions. La mise en lumière et l'analyse des formes de résistance et de contestation mises en œuvre par les migrants nous ont semblé fondamentales dans un second temps de la recherche, afin de restituer le juste équilibre entre mécanismes de contrôle et autonomie du sujet, et de comprendre, dans leurs ambivalences et leurs tensions, les multiples ressorts du pouvoir et de la subjectivation. Cette entreprise de décentrement a emprunté différents canaux et s'est basée sur plusieurs méthodes (observations courtes ou participantes ; multiplication d'entretiens ; organisation d'évènements, etc.) dans une perspective de triangulation des sources et des techniques de recueil des données : nous en rapportons ici quelques unes.

Tout d'abord, nous avons essayé de mettre en lumière les formes de mobilisation collectives mises en œuvre par les migrants. Deux évènements ont fait date récemment: d'une part, la naissance d'un mouvement anti-raciste maltais initié par des migrants établis à Malte depuis quelques années, qui s'impose progressivement sur la scène publique et porte une revendication de droit à l'installation et d'accès aux services élémentaires. D'autre part, les révoltes contre les conditions de rétention, qui ont été particulièrement violentes cet été, notamment dans celui de Safi, un ancien camp militaire converti en centre de rétention ${ }^{11}$. Ces deux mobilisations collectives, l'une plus structurée et l'autre ponctuelle, l'une plus pacifique et l'autre plus violente, ont une caractéristique commune : loin de fonctionner en boîte noire, elles portent la trace des mobilisations précédentes et font écho aux multiples révoltes méditerranéennes. Les militants du mouvement anti-raciste rappellent volontiers la révolte de Rosarno, village de Calabre où, en décembre 2008 et en janvier 2010, les ouvriers agricoles africains ont décidé de se rebeller contre leur condition (Outis, 2011) ; tandis que les révoltes des centres de rétention renvoient aux émeutes qui ont lieu parallèlement en Italie et en Tunisie, et dont les migrants se tiennent informés grâce aux dispositifs de télécommunication. La Méditerranée devient alors la caisse de résonnance de subjectivités politiques "hors citoyenneté " qui, relayées par les nouvelles technologies et les circulations migrantes, donnent une ampleur et une consistance nouvelles aux mouvements et initiatives maltais. Ils contribuent à définir des espaces de résistance et de projet qui vont bien au-delà des frontières maltaises.

Dans cette tentative de mise en lumière des paroles et des actes des migrants, un moment a été important dans notre trajectoire de recherche : à l'occasion d'un colloque, nous avons organisé grâce à l'aide de deux collègues fortement engagées

\footnotetext{
${ }^{11}$ http://www.timesofmalta.com/articles/view/20110816/local/safi-migrants-start-fire-in-protest.380542. Déjà une révolte avait éclaté en mars 2009 dans le centre de Safi.
} 
dans la défense des droits des migrants $^{12}$ à Malte une table-ronde permettant, pendant deux heures, de donner la parole aux migrants. II s'agissait pour nous, plutôt que d'organiser la table-ronde habituelle mettant en scène les différents " experts " de la question (ONG, HCR, Agence Européenne pour l'asile, etc.), de donner l'opportunité à six migrants de parler à la première personne des difficultés qu'ils avaient rencontrées à Malte, des liens qu'ils avaient pu établir avec la société locale, ainsi que de leur souhaits et projets pour l'avenir. L'audience était a priori conquise puisqu'il s'agissait avant tout d'universitaires et de travailleurs sociaux. Cependant, l'évènement a pris une tournure intéressante: d'abord parce que les interactions entre les migrants et le public, essentiellement maltais, furent franches, dynamiques et passionnées, mais aussi parce qu'elles permirent d'aborder des questions qui n'avaient que rarement été ouvertement discutées au-delà du petit cercle des militants. La question du "fardeau » représenté par les migrants, par exemple, qui est un lieu commun à Malte - les migrants africains représenteraient un fardeau pour la société, et les autres Etats européens devraient s'engager à aider Malte pour prendre leur part du fardeau (burden sharing) - a fait l'objet d'une déconstruction systématique de la part des migrants. Goitom Yosef, un travailleur social éthiopien, expliqua, par exemple, à quel point il ne représentait guère un fardeau pour le pays à l'exception du moment où il était en détention, mettant ainsi en évidence l'absurdité de la politique de détention maltaise : "Politicians speak of burden sharing ... but I'm not a burden. I have a job, I pay my taxes. I contribute to society. Maybe I was a burden on the state when I was in detention, but not anymore" (GY). Un ouvrier somalien, Abishi Abdala, insista sur le fait que l'île constituait pour lui un lieu d'installation et de projet, contestant ainsi l'image largement répandue selon laquelle les migrants se seraient que des oiseaux de passage en transit sur l'île : "People believe that migrants use Malta as a stepstone. It is not true. I am 32, I have a permanent job in Malta and I am proud of that. I want to stay in Malta But I have no sense of belonging. This is sad. We can have a sense of belonging only if we are allowed to stay permanently" (AA).

Tout en soulevant l'ambivalence de ses sentiments vis-à-vis de l'île, Abishi Abdala évoqua la question du droit au regroupement familial, qui est encore impossible à Malte, y compris pour les migrants en situation régulière. L'évènement eut un retentissement dans la presse quotidienne ${ }^{13}$, générant des réactions certes souvent xénophobes mais parfois aussi empathiques, ce qui là aussi, est une rareté dans le contexte maltais. Pour nous, cette table-ronde a constitué une remarquable occasion de recueil de témoignages, même si, bien sûr, les participants à la table ronde n'étaient aucunement représentatifs de tous les migrants (Milhaud, 2006) : il s'agissait

\footnotetext{
12 Il s'agit de Maria Pisani et Céline Warnier de Wailly qui elles-mêmes développent, dans le cadre de leurs thèses de doctorat, des approches critiques vis-à-vis du phénomène migratoire maltais.

${ }^{13} \mathrm{http}$ ://www.timesofmalta.com/articles/view/20111213/local/-We-are-not-bad-.398150
} 
d'intervenants « qualifiés pour parler »-singulièrement des hommes, à une exception près - et prêts à se confronter à l'arène publique.

En parallèle, nous avons donc essayé d'orienter nos recherches vers d'autres méthodes et d'autres populations, moins "parlantes " et moins visibles, souvent stéréotypées et décrites comme particulièrement vulnérables, afin de restituer, tant que faire se peut, l'hétérogénéité de la population migrante présente à Malte. Le travail que nous avons mené sur le rôle du corps des femmes s'est inscrit dans cette préoccupation. Ce n'est plus tant de donner la parole en public ou d'observer les mobilisations collectives qu'il s'est agi, mais de passer du temps avec les femmes résidant en centre d'accueil, de partager leur quotidien et de discuter à bâtons rompus avec elles.

Les femmes, en effet, s'investissent moins dans la sphère publique: de par leur invisibilité, elles sont souvent perçues uniquement comme des victimes. Ces moments d'observation participante nous ont permis de prendre conscience, en opposition avec " ceux qui prennent la parole ", de l'importance du corps pour ces femmes, dans la construction, éminemment politique, de leur subjectivité migrante (Bayart, Warnier, 2004). En observant le corps des femmes, à travers les techniques, postures, mises en scènes et usages qu'elles en faisaient, et en les écoutant raconter les transformations qu'il subissait au fur et à mesure de leur trajectoire migratoire, nous avons pu saisir en effet combien il constitue une bonne entrée à la compréhension d'une " autonomie en tension ", dans un contexte de durcissement des politiques migratoires (Schmoll, 2011). Si celui-ci est souvent violenté et affecté par la maladie et l'épuisement tout au long de la trajectoire migratoire, le corps des femmes peut également donner la vie : les enfants naissent en mer, dans le désert, à l'arrivée en Europe. Etre enceinte et enfanter, c'est alors s'approprier son corps pour aller de l'avant. Même quand ces enfants sont le fruit de violences, ils sont vécus par les femmes rencontrées comme une richesse. Les enfants comme force, bien loin des discours moralisateurs selon lesquels la natalité des femmes migrantes constituerait un frein à leur mobilité et à leur émancipation ... Les travaux sur l'éthique du care montrent bien d'ailleurs comment l'autonomisation est avant tout relationnelle et peut passer par le soin des autres (Laugier, 2009). De ce point de vue, donner la vie en Europe ou sur la route de l'Europe, c'est pour ces femmes déjà opérer une forme de re-territorialisation, poser des ancrages.

Mais le soin de soi ne passe pas nécessairement par l'agrandissement de la famille et d'autres femmes racontent, à l'inverse, combien la contraception ou l'abstinence peuvent les aider à s'en sortir telle Dunia, une jeune femme somalienne de 22 ans qui ne veut pas se marier avec son fiancé car avoir des enfants l'empêcherait de poursuivre des études qu'elle souhaite effectuer aux Etats-Unis. La technique du corps investit également les routines - telles que les routines religieuses, quand la prière rythme de longues journées d'ennui en centre d'accueil et donne la force d'aller de 
l'avant - ou encore les moments de fête - telle que celle organisée par cette femme qui, apprenant sa relocalisation imminente aux Etats-Unis, décide de se teindre les cheveux en blond.

En prenant le corps des femmes à témoin, en observant les techniques et tactiques mises en place par celles-ci ainsi que leurs récits sur les transformations de leur corps en migration, nous souhaitions montrer qu'il est - certes - le support de souffrances et d'une vulnérabilisation accrue, le lieu d'incorporation de politiques migratoires sexuées et de techniques de contrôle des flux - que ce soit à travers le contrôle sur la reproduction et la sexualité des migrantes ou à travers le traçage biométrique, mais qu'il est aussi le lieu de guérisons ou de plaisirs et de stratégies de résistance permettant une reconstruction identitaire.

\section{Conclusion. Repenser la posture du géographe pour repenser les migrations}

La dénonciation et l'engagement empêchent-ils la prise de distance nécessaire à une recherche scientifique ? Est-il possible tour à tour de dénoncer la situation dont sont victimes les migrants relégués aux marges de l'Europe et de mettre en lumière leurs initiatives et actions de résistance ? II nous semble que les deux temps de la recherche qui viennent d'être décrits ne peuvent être dissociés, et que les chercheur(-e)s ne peuvent prétendre faire l'économie, dans le cadre d'une démarche scientifique, de leur engagement. De ce point de vue, notre travail ne se résume pas uniquement à restituer nos émotions et notre colère face à la situation des migrants - ce qui reviendrait à s'engager dans une posture totalement relativiste. II n'est pas non plus uniquement le produit de ce qu'expriment nos interlocuteurs dont nous ne serions que de simples "porte-parole " (Bertrand, 2007, p. 278) mais a aussi et surtout pour ambition de mettre en lumière certains mécanismes de contrôle, quitte à les éprouver parfois directement. En même temps, écouter et observer les autres au quotidien et dans diverses situations nous a permis de faire émerger leurs multiples individualités, subjectivités et projets, loin de l'image figée et homogène de victime (au mieux) ou d'opportuniste (au pire) qui caractérise souvent les migrants africains présents sur l'île. Notre travail s'est donc réalisé à plusieurs voix, d'abord celles des deux chercheures que nous sommes et avec tout le pouvoir que peut conférer ce statut (pouvoir d'énonciation, d'organisation, de dénonciation), mais aussi celles des multiples interlocuteurs que nous avons rencontrés - migrants et autres - et qui ne sont pas sur le même pied d'égalité. Cette recherche est donc le produit de cette toile polyphonique le plus souvent discordante qui ne cesse de se tisser et de se retisser au fur et à mesure de l'évolution de la situation migratoire en Méditerranée et à Malte en particulier. 
Concilier engagement et démarche scientifique ne peut se faire qu'au prix de contorsions, d'adaptations, d'arrangements multiples. Il est absolument nécessaire de rendre compte de ces oscillations et discordances, de situer et de contextualiser notre travail de géographe : loin d'être un exercice narcissique et vain, la réflexivité sur les conditions du recueil des données discursives et observationnelles, la contextualisation de notre statut de chercheur-e engagé-e, la triangulation des sources et des méthodes sont autant de voies nécessaires dans la quête de l' « approximative rigueur " qui commande toute recherche (de Sardan, 2008). Au-delà des aspects strictement factuels, le dévoilement des coulisses de notre recherche à Malte a eu une réelle vertu heuristique : repenser le positionnement, le rôle et la voix du géographe est une condition sine qua non pour repenser les migrations (Green, 2002).

\section{Bibliographie}

Agier M. (1997), Anthropologues en dangers : l'engagement sur le terrain, Paris, Éd. Jean-Michel Place, 128 p. (Cahiers de Gradhiva, $n^{\circ} 30$ ).

Agier M. (2008), Gérer les indésirables. Des camps de réfugiés au gouvernement humanitaire, Paris, Flammarion, $350 \mathrm{p}$.

Bayart J.F, Warnier J.P. (2004), Matière à politique. Le pouvoir, les corps et les choses, Paris, Karthala, 255 p.

Bertrand R. (2007), "Faire parler les subalternes ou le mythe du dévoilement ", in SMOUTS M.-C (dir.)., La situation postcoloniale, Paris, Presses de Sc. Po, pp. 276-284.

Brachet J. (2006), « Du questionnement éthique à l'engagement politique: un cheminement nécessaire ? ", http://eegeosociale.free.fr/rennes2006, 6p.

Collyer M. (2007), Undocumented sub-Saharan migrants in Morocco, Copenhagen, Danish Institute for International Studies, pp. 129-146.

Collyer M., Duvelle F., de Haas H. (2010), "Critical approaches to transit migration", Population, space and place, vol. 18 (4), pp.407-414.

Cruse R. (2011), Géopolitique d'une périphérisation du bassin caribéen, Presses de I'Université du Québec, 153 p.

De Luze H. (1997) L'ethnométhodologie, Paris, Anthropos, 111 p.

Olivier de Sardan J-P. (2008), La rigueur du qualitatif, Louvain, Bruylant, 366 p. 
Fassin D. (1998), "L'anthropologie entre engagement et distanciation. Essai de sociologie des recherches en sciences sociales sur le sida en Afrique", in Charles Becker, Jean-Pierre Dozon, Christine Obbo et Moriba Touré (dir.), Vivre et penser le sida en Afrique, Paris : IRD/Karthala/Codesria, 1998, p. 41-66.

Grignon C. et Passeron J.-C. (1989), Le savant et le populaire. Misérabilisme et populisme en sociologie et en littérature, Paris, Gallimard, $260 \mathrm{p}$.

Green N. (2008), Repenser les migrations, Paris, PUF, 140 p.

Laugiers S. (2009), "L'autonomie et le souci du particulier », in LAUGIER S., JOUAN M. (dir.), Comment penser l'autonomie ? Entre compétences et dépendances, Paris, PUF, pp. 389-407.

Lefort I. (2010), "A quel prix la géographie est-elle soluble dans l'éthique ?", Géographie et cultures, $\mathrm{n}^{\circ} 74, \mathrm{pp} .11-25$.

Milhaud O. (2006), " La géographie, la prison et l'éthique : prestige et vertige de la justice $"$, http://eegeosociale.free.fr/rennes2006, 4 p.

Morelle M. Et Ripoll F. (2009), "Les chercheur-es face aux injustices : l'enquête de terrain comme épreuve éthique ", Annales de Géographie, n 665-666, pp. 157-168.

Outis! (2011), Numéro spécial "Révoltes Migrantes ", Outis! Revue de philosophie (post-)européenne, Milan, Mimesis.

Racine J.-B. (2010), "Géographie, éthique et valeurs: invitation à la réflexion et à I'action ", Géographie et cultures, n74, pp. 27-42.

Schmoll C. (2011), "Le corps des autres dans l'Europe des frontières ", Outis. Revue de philosophie (post-)européenne, 1, pp. 121-128.

Spivak G. (1985), "Can the Sulbaltern speak ? », in C. NELSON C., GROSSBERG L. (dir.), Marxism and the Interpretation of Culture, Chicago, University of Illinois press, pp. 271313.

Staszak J.-F. (1997), « Dans quel monde vivons-nous ? Géographie, phénoménologie et ethnométhodologie ", Staszak J.-F. (dir.), Les discours du géographe, Paris: L'harmattan, pp. 13-38.

Widdowfield R. (2000), "The place of emotions in academic research », Area, 32, 2, pp. 199-208.

Zeneidi D. (2002), Les SDF et la ville, géographie du savoir-survivre, Paris, Bréal, 288 p. 
Carnets de géographes, $\mathrm{n}$ - 4, septembre 2012

Rubrique Carnets de terrain

Zeneidi D. (2010), "Comment on balade le chercheur au sens propre et figuré » http://terrferme.hypotheses.org/363, billet posté le 8 juin 2010. 